\title{
Decisions following reading and bias in recall
}

\author{
VICKY TIMME, ALICE CORKILL, and JOHN A. GLOVER \\ University of Nebraska, Lincoln, Nebraska
}

\begin{abstract}
The role of decision making in biasing recall of prose was examined. Both making a decision subsequent to reading and encountering an experimenter-provided decision resulted in recall biased in the direction of the decision. Conditions in which subjects made their own decisions, however, resulted in greater levels of decision-congruent recall than did conditions in which the experimenters furnished decisions to subjects. In addition, the level of recall of incongruent information in the subject-made decisions conditions was as high as the level of congruent information observed in the experimenter-provided decisions conditions. A memory-restructuring model, suggested by Dellarosa and Bourne (1984), accounts for the results if it is true that greater elaboration of both decision-congruent and decision-incongruent information during subject-made decisions occurs.
\end{abstract}

Recently, Dellarosa and Bourne (1984) observed that the provision of additional information subsequent to reading had essentially the same effect on bias in recall as did decision making. They argued that the decision-making process itself had little impact on how information about the passage was stored. They suggested that the decision served the same function as a statement given readers subsequent to reading; that is, either the decision itself or the statement was stored as a focal point for the information retained in memory related to the passage.

Although it seems clear from Dellarosa and Bourne's (1984) work that both decisions and statements given after reading bias recall, it is unclear whether the processes involved in obtaining these biases are the same. Several studies have shown that decision making increases the amount of text material recalled relative to other procedures that do not involve decision making on the part of readers (e.g., Benton, Glover, \& Bruning, 1983; Benton, Glover, Monkowski, \& Shaughnessy, 1983; Glover, Bruning, \& Plake, 1982). It may very well be that statements provided after reading bias recall by reducing the potential that decision-incongruent information will be available at the time of recall. In terms of decisions, however, it seems that an alternative explanation is possible; that is, biases may result not from the attenuated availability of decision-incongruent information at the time of recall, but rather through an increase in the amount of decision-congruent information available.

The current study examined the effects of decision making and the provision of statements subsequent to reading on biases in readers' recall. Five conditions were included: subject-made positive decisions (SP), subjectmade negative decisions (SN), experimenter-provided positive intervening information (EP), experimenterprovided negative intervening information (EN), and con-

Requests for reprints should be sent to John A. Glover, Department of Educational Psychology, University of Nebraska, Lincoln, NE 68588-0440. Copies of the stimulus materials are available on request. trol. Both positive and negative options were included because of the possibility that they might differentially influence recall.

\section{METHOD}

\section{Subjects, Setting, and Materials}

Ninety undergraduate volunteers participated for course credit. Testing took place in groups of 6-8. Subjects encountered a 25-sentence paragraph about the foreign policy of a mythical nation, Mala. The first sentence served as an unscored introduction; the remaining 24 sentences each contained one idea unit. Twelve sentences described Mala's policy as favoring the West. Twelve described its policy as favoring the Soviet Union.

\section{Procedure}

Subjects were randomly assigned to four conditions. The SN and SP conditions were treated as one condition in the original assignment of subjects to conditions, and twice as many subjects were assigned to this combined condition as to the others. Later, on the basis of the decisions made by subjects, subjects in this condition were separated into the SN and SP conditions.

All subjects were asked to read and study carefully for a posttest over the material. No mention was made of decisions or postreading intervening information. Upon completion, they performed an interpolated task (answering 50 simple addition problems) and then completed a free recall posttest. Control subjects were given no further instructions or materials. Subjects in each of the other conditions turned the page when they finished. The second page required subjects in the subject-made decisions condition to decide in writing whether Mala's foreign policy favored the West or the Soviet Union. Subjects in the EP and EN conditions encountered the experimenter-provided intervening information on the second page. EP subjects were told that Mala's policy favored the West. EN subjects were told that Mala's policy favored the Soviet Union.

\section{RESULTS}

Subjects' decisions in the subject-made decision condition were examined, and subjects were sorted into the SP (favored the West) and SN (favored the Soviet Union) conditions. Subjects' protocols were then scored by two independent raters for gist recall $(r=.98)$. In order to examine the potential for bias in recall, we performed a 
repeated measures analysis of variance employing conditions as the independent variable and positive and negative recall as the repeated measures. In examining the results of this analysis, we observed a significant type of recall (positive or negative) $\times$ treatment condition interaction $[F(4,85)=27.70, p<.01, M S e=53.35]$. This significant interaction indicated that there were different levels of positive and negative recall across conditions. In order to follow up on this result, we performed univariate analyses of variance on each of the dependent variables. A significant effect was observed among conditions on positive recall $[F(4,85)=9.45, p<.01$, $M S \mathrm{e}=4.21]$. Similarly, a significant effect was identified among conditions on negative recall $[F(4,85)=$ $11.87, p<.01, M S e=4.35$ ]

The univariate analyses were then followed up via the Tukey HSD procedure $(\alpha=.05)$. In terms of positive recall, significantly more positive ideas were recalled in the SP condition (mean $=9.38, S D=1.36$ ) than in any other condition. No significant difference in recall of positive ideas was observed among the control (mean $=6.72$, $S D=2.27)$, the EP $($ mean $=7.72, S D=2.08)$, and the $\mathrm{SN}$ (mean $=7.15, S D=2.16$ ) conditions. Each of these conditions, however, resulted in significantly greater recall of positive ideas than did the EN condition (mean $=5.17, S D=2.18$ ). No other contrasts reached significance.

When negative recall was examined, subjects in the SN condition (mean $=9.35, S D=2.21$ ) recalled significantly more negative ideas than did subjects in any other condition. No significant difference was observed among the control (mean $=6.39, S D=2.12$ ), the EN (mean $=7.77, S D=1.63$ ), and the $\mathrm{SP}$ (mean $=7.25, S D=$ 2.14 ) conditions. Subjects in the EP condition (mean = $5.50, S D=2.26$ ) recalled significantly fewer negative ideas than did subjects in the EN and SP conditions. No other contrasts reached significance.

A second analysis focused on the recall of congruent and incongruent ideas. For example, in the EP condition, recall of positive ideas was considered congruent recall, as was the recall of negative ideas in the $\mathrm{SN}$ condition. Then, as we were equally interested in both the congruent and incongruent recall variables, we performed separate univariate analyses on each variable, excluding the control condition from the analysis. A significant difference among conditions was observed on recall of congruent ideas $[F(3,68)=6.28, p<.01, M S e=3.52]$. Similarly, a significant difference was observed among conditions on the recall of incongruent ideas $[F(3,68)=4.43$, $p<.01, M S e=4.77]$.
The univariate analyses were then followed up via the Tukey HSD procedure $(\alpha=.05)$. No significant difference was observed on the recall of congruent ideas between the SP condition (mean $=9.38, S D=1.36$ ) and the $S N$ condition $($ mean $=9.35, S D=2.21)$. Subjects in each of these conditions, however, recalled significantly more congruent ideas than did subjects in either the EP condition (mean $=7.72, S D=2.08$ ) or the EN condition (mean $=7.77, S D=1.63$ ). No other contrasts reached significance.

When recall of incongruent ideas was considered, no significant difference was observed between the SP condition (mean $=7.25, S D=2.14$ ) and the $S N$ condition (mean $=7.15, S D=2.16$ ). Subjects in each of these two conditions, however, recalled significantly more incongruent ideas than did subjects in either the EP condition (mean $=5.50, S D=2.26$ ) or the EN condition (mean $=5.17, S D=2.18$ ). No other contrasts reached significance.

\section{DISCUSSION}

Patterns of bias similar to those observed by Dellarosa and Bourne (1984) appeared in the current results; that is, both decision making and the imposition of experimenter-provided information biased recall as contrasted to the control condition. Subjects in the SN and SP conditions clearly recalled more congruent information, however, than did subjects in the EP or EN conditions. In addition, the level of recall of incongruent information in the SN and SP conditions was as high as the level of congruent information observed in the EP and EN conditions. Apparently, the decision-making process itself does have an effect, one that seems to increase the overall levels of recall of both decision-congruent and decision-incongruent recall. The model described by Dellarosa and Bourne (1984) can easily account for the current results if it is true that the process of decision making itself enhances recall through more elaborative encoding of both decision-congruent and decision-incongruent information (Ross, 1981).

\section{REFERENCES}

Benton, S. L., Glover, J. A., \& Bruning, R. H. (1983). The effect of number of decisions on prose recall. Journal of Educational Psychology, 75, 382-390.

Benton, S. L., Glover, J. A., Monkowski, P. G., \& Shaughnessy, M. (1983). Decision difficulty and recall of prose. Journal of Educational Psychology, 75, 727-742.

Dellarosa, D., \& Bourne, L. E. (1984). Decisions and memory: Differential retrievability of consistent and contradictory evidence. Journal of Verbal Learning \& Verbal Behavior, 23, 669-682.

Glover, J. A., Bruning, R. H., \& Plake, B. S. (1982). Distinctiveness of encoding and recall of text materials. Journal of Educational Psychology, 74, 522-524.

Ross, B. H. (1981). The more the better? Number of decisions as a determinant of memorability. Memory \& Cognition, 9, 23-33.

(Manuscript received for publication September 19, 1986.) 